\title{
DUAL TRANSLATION SURFACES IN THE THREE DIMENSIONAL SIMPLY ISOTROPIC SPACE $\square_{3}^{1}$
}

\author{
MOHAMD SALEEM LONE AND MURAT KEMAL KARACAN
}

\begin{abstract}
In this paper, we study the dual translation surfaces in three dimensional simply isotropic space. We give classification of dual translation surface with constant dual isotropic mean curvature or constant dual isotropic Guassian curvature.
\end{abstract}

\section{Introduction}

Projective spaces enjoy a principle of duality, for example in projective 3-spaces, points are dual to planes and vice versa, straight lines are dual to straight lines and inclusions are reversed. However, duality cannot be applied to metric quantities of Euclidean geometry. This is different in isotropic geometry, which have a metric duality which may be realized by the polarity with respect to the isotropic unit sphere

$$
\Sigma: z=\frac{1}{2}\left(x^{2}+y^{2}\right)
$$

It maps a point $\mathbf{p}=\left(p_{1}, p_{2}, p_{3}\right)$ to the plane $P$ with equation $z=p_{1} x+p_{2} y-p_{3}$. Points $\mathbf{p}$, $\mathbf{q}$ with i-distance $d$ are mapped to planes $P, Q$ with i-angle $d$ and vice versa. Parallel points correspond in the duality to parallel planes.

A surface $\mathbf{x}: z=h(u, v)$, seen as a set of contact elements (points plus tangent planes) corresponds to a surface $\mathbf{x}^{*}$, parameterized by

$$
\left\{\begin{array}{l}
x^{*}=h_{u}(u, v) \\
y^{*}=h_{v}(u, v) \\
z^{*}=u h_{u}(u, v)+v h_{v}(u, v)-h(u, v) .
\end{array}\right.
$$

Contact elements along isotropic principal curvature lines of $\mathbf{M}$ and $\mathbf{M}^{*}$ correspond in the duality. Note that $\mathbf{M}^{*}$ may have singularities which correspond to parabolic surface points of

Received May 8, 2017, accepted November 14, 2017.

2010 Mathematics Subject Classification. Primary 53A35, 53B30, 53A40.

Key words and phrases. Dual surface, simply isotropic space, translation surface.

Corresponding author: Mohamd Saleem Lone. 
$\mathbf{M}(\mathbf{K}=0)$. This is reflected in the following relations between the isotropic curvature measures of dual surface pairs:

$$
\mathbf{K}^{*}=\frac{1}{\mathbf{K}}, \mathbf{H}^{*}=\frac{\mathbf{H}}{\mathbf{K}},
$$

where $\mathbf{H}(\mathbf{K})$ is the isotropic mean(Gaussian) curvature and $\mathbf{H}^{*}\left(\mathbf{K}^{*}\right)$ is the dual isotropic mean (Gaussian) curvature. Thus, the dual isotropic minimal surface is also isotropic minimal ([7, $6])$.

In this paper, we extended the notion of duality to translation surfaces in isotropic spaces and obtain the classification results for dual isotropic curvatures of translation surfaces.

\section{Preliminaries}

Isotropic geometry is based on the following group $\mathbf{G}_{6}$ of affine transformations $(x, y, z) \rightarrow$ $\left(x^{\prime}, y^{\prime}, z^{\prime}\right)$ in $\mathbb{R}^{3}$,

$$
\left.\begin{array}{l}
x^{\prime}=a+x \cos \theta-y \sin \theta \\
y^{\prime}=b+x \sin \theta+y \cos \theta \\
z^{\prime}=c+c_{1} x+c_{2} y+z
\end{array}\right\}
$$

where $a, b, c, c_{1}, c_{2}, \theta \in \mathbb{R}$. Such affine transformations are called isotropic congruence transformations or isotropic motions . We see that isotropic motions appear as Euclidean motions (a translation and a rotation) in the projection onto the $x y$-plane. The result of this projection, $P=(x, y, z) \rightarrow P^{\prime}=(x, y, 0)$ is called the "top view" $([7,6,12])$. Hence, an isotropic motion is composed of a Euclidean motion in the $x y$-plane and an affine shear transformation in the $z$-direction.

On the other hand, the isotropic distance of two points $P=\left(x_{1}, y_{1}, z_{1}\right)$ and $Q=\left(x_{2}, y_{2}, z_{2}\right)$ is defined as the Euclidean distance of the top views, i.e.,

$$
d(P, Q)_{i}=\sqrt{\left(x_{1}-x_{2}\right)^{2}+\left(y_{1}-y_{2}\right)^{2}}
$$

Let $X=\left(x_{1}, y_{1}, z_{1}\right)$ and $Y=\left(x_{2}, y_{2}, z_{2}\right)$ be vectors in $\square_{3}^{1}$. The isotropic inner product of $X$ and $Y$ is defined by

$$
\langle X, Y\rangle_{i}= \begin{cases}z_{1} z_{2}, & \text { if } x_{i}=y_{i}=0, \\ x_{1} x_{2}+y_{1} y_{2}, & \text { if otherwise }\end{cases}
$$

We call a vector of the form $X=(0,0, z)$ in $\square_{3}^{1}$ an isotropic vector, and a non-isotropic vector otherwise. Consider a $C^{r}$-surface $\mathbf{M}, r \geq 1$, in $\rrbracket_{3}^{1}$ parameterized by

$$
\mathbf{x}(u, v)=(x(u, v), y(u, v), z(u, v)) .
$$


A surface $\mathbf{M}$ immersed in $\square_{3}^{1}$ is called admissible if it has no isotropic tangent planes. We restrict our framework to admissible regular surfaces $([1,9,7,12])$.

For such a surface, the coefficients $E, F, G$ of its first fundamental form are calculated with respect to the induced metric and the coefficients $L, M, N$ of the second fundamental form, with respect to the normal vector field of a surface which is always completely isotropic. The first and the second fundamental form of $\mathbf{M}$ are defined by

$$
\begin{gathered}
I=E d u^{2}+F d u d v+G d v^{2}, \\
I I=L d u^{2}+M d u d v+N d v^{2},
\end{gathered}
$$

where

$$
\begin{aligned}
& E=\left\langle\mathbf{x}_{u}, \mathbf{x}_{u}\right\rangle_{i}, F=\left\langle\mathbf{x}_{u}, \mathbf{x}_{v}\right\rangle_{i}, G=\left\langle\mathbf{x}_{v}, \mathbf{x}_{v}\right\rangle_{i}, \\
& L=\frac{\operatorname{det}\left(\mathbf{x}_{u}, \mathbf{x}_{v}, \mathbf{x}_{u u}\right)}{\sqrt{E G-F^{2}}}, M=\frac{\operatorname{det}\left(\mathbf{x}_{u}, \mathbf{x}_{v}, \mathbf{x}_{u v}\right)}{\sqrt{E G-F^{2}}}, N=\frac{\operatorname{det}\left(\mathbf{x}_{u}, \mathbf{x}_{v}, \mathbf{x}_{v v}\right)}{\sqrt{E G-F^{2}}} .
\end{aligned}
$$

Since $E G-F^{2}>0$, for the function in the denominator we often put $W^{2}=E G-F^{2}$. The isotropic unit normal vector field is given by $\mathbf{U}=(0,0,1)$. The isotropic Gaussian curvature $\mathbf{K}$ and the isotropic mean curvature $\mathbf{H}$ are defined by

$$
\mathbf{K}=\frac{L N-M^{2}}{E G-F^{2}}, 2 \mathbf{H}=\frac{E N-2 F M+G L}{E G-F^{2}} .
$$

The surface $\mathbf{M}$ is said to be isotropic flat (resp. isotropic minimal) if $\mathbf{K}$ (resp. $\mathbf{H}$ ) vanishes $([1,3,8,9,10,7])$.

We confine our discussion to regular surfaces $\mathbf{x}$ without isotropic tangent planes. Thus, we may write in explicit form;

$$
\mathbf{x}: z=h(u, v) \text {. }
$$

\section{Translation Surfaces in $\rrbracket_{3}^{1}$}

In order to describe the isotropic analogues of translation surfaces of constant curvatures, we consider translation surfaces obtained by translating two planar curves $\alpha$ and $\beta$. The local surface parametrization is given by

$$
\mathbf{x}(u, v)=\alpha(u)+\beta(v) .
$$

Since there are, with respect to the absolute figure, different types of planes in $\square_{3}^{1}$, there are in total three different possibilities for planes that contain translated curves: the translated curves can be curves in isotropic planes (which can be chosen, by means of isotropic motions, as $y=0$, resp. $x=0)$; or one curve is in a non-isotropic plane $(z=0)$ and one curve in an isotropic plane $(y=0)$; or both curves are curves in non-isotropic perpendicular planes 
( $y-z=\pi$, resp. $y+z=\pi$ ). Therefore, the obtained translation surfaces allow the following parametrizations ([9]):

Type 1: The surface $\mathbf{M}$ is parametrized by

$$
\mathbf{x}(u, v)=(u, v, f(u)+g(v))
$$

and the translated curves are $\alpha(u)=(u, 0, f(u)), \beta(v)=(0, v, g(v))$. The dual isotropic Gaussian and dual isotropic mean curvature of dual translation surfaces of Type 1 are given by

$$
\mathbf{K}^{*}=\frac{1}{f^{\prime \prime}(u) g^{\prime \prime}(v)}, \mathbf{H}^{*}=\frac{f^{\prime \prime}(u)+g^{\prime \prime}(v)}{2 f^{\prime \prime}(u) g^{\prime \prime}(v)} .
$$

Type 2: The surface $\mathbf{M}$ is parametrized by

$$
\mathbf{x}(u, v)=(u, f(u)+g(v), v)
$$

and the translated curves are $\alpha(u)=(u, f(u), 0), \beta(v)=(0, g(v), v)$. In order to obtain admissible surfaces, $g^{\prime}(v) \neq 0$ is assumed (i.e. $g(v) \neq$ constant). The dual isotropic Gaussian and dual isotropic mean curvature of dual translation surfaces of Type 2 are given by

$$
\mathbf{K}^{*}=\frac{g^{\prime^{4}}(u)}{f^{\prime \prime}(u) g^{\prime \prime}(v)}, \mathbf{H}^{*}=-\frac{f^{\prime \prime}(u) g^{\prime^{3}}(\nu)+g^{\prime}(\nu) g^{\prime \prime}(\nu)\left(1+f^{\prime^{2}}(u)\right)}{2 f^{\prime \prime}(u) g^{\prime \prime}(v)} .
$$

Type 3: The surface $\mathbf{M}$ is parametrized by

$$
\mathbf{x}(u, v)=\frac{1}{2}(f(u)+g(v), u-v+\pi, u+v),
$$

and the translated curves are

$$
\alpha(u)=\frac{1}{2}\left(f(u), u+\frac{\pi}{2}, u-\frac{\pi}{2}\right), \beta(v)=\left(g(v), \frac{\pi}{2}-v, \frac{\pi}{2}+v\right) .
$$

In order to obtain admissible surfaces, $f^{\prime}(u)+g^{\prime}(v) \neq 0$ is assumed (i.e. $f^{\prime}(u) \neq-g^{\prime}(v)=$ $a=$ constant). The dual isotropic Gaussian and dual isotropic mean curvature of dual translation surfaces of Type 3 are given by

$$
\begin{aligned}
& \mathbf{K}^{*}=\frac{\left(f^{\prime}(u)+g^{\prime}(v)\right)^{4}}{16 f^{\prime \prime}(u) g^{\prime \prime}(v)}, \\
& \mathbf{H}^{*}=\frac{\left(f^{\prime}(u)+g^{\prime}(v)\right)\left(f^{\prime \prime}(u)\left(1+g^{\prime^{2}}(v)\right)+g^{\prime \prime}(v)\left(1+f^{\prime^{2}}(u)\right)\right)}{16 f^{\prime \prime}(u) g^{\prime \prime}(v)} .
\end{aligned}
$$




\section{Dual Isotropic Curvatures of the Dual Translation Surfaces of Type 1}

Suppose the dual mean curvature $\mathbf{H}^{*}$ of the dual translation surface of Type 1 in $\mathbb{1}_{3}^{1}$ vanishes identically, from (3) we have

$$
f^{\prime \prime}+g^{\prime \prime}=0
$$

Then we have

or

$$
\left\{\begin{array}{l}
f(u)=\left(c_{1} u+c_{2}\right), \\
g(v)=\left(c_{3} v+c_{4}\right),
\end{array}\right.
$$

$$
\left\{\begin{array}{l}
f(u)=\left(p \frac{u^{2}}{2}+c_{1} u+c_{2}\right), \\
g(v)=\left(-p \frac{v^{2}}{2}+c_{3} v+c_{4}\right),
\end{array}\right.
$$

where $p, c_{i} \in \mathbb{R}$.

Now, we consider dual translation surface of Type 1 with non-zero dual constant mean curvature in simply isotropic 3 -space $\square_{3}^{1}$. If the mean curvature $\mathbf{H}^{*}$ is non-zero constant, from (3), we have

$$
2 \mathbf{H}^{*}=\frac{f^{\prime \prime}(u)+g^{\prime \prime}(v)}{f^{\prime \prime}(u) g^{\prime \prime}(v)} .
$$

From (11), we have

or

$$
2 \mathbf{H}^{*}-\frac{1}{g^{\prime \prime}(v)}=\frac{1}{f^{\prime \prime}(u)},
$$

$$
2 \mathbf{H}^{*}-\frac{1}{f^{\prime \prime}(u)}=\frac{1}{g^{\prime \prime}(v)} \text {. }
$$

Here $u$ and $v$ are independent variables, so each side of (12) is equal to a constant, call it $p$. Hence, the two equations are

$$
2 \mathbf{H}^{*}-\frac{1}{g^{\prime \prime}(\nu)}=p=\frac{1}{f^{\prime \prime}(u)}
$$

Its general solution is

$$
\left\{\begin{array}{l}
f(u)=c_{1} u+c_{2}+\frac{u^{2}}{2 p}, \\
g(v)=c_{3} v+c_{4}+\frac{v^{2}}{2\left(2 \mathbf{H}^{*}-p\right)}
\end{array}\right.
$$

for some constants $c_{i} \neq 0$ and $p \neq 0$.

Theorem 4.1. Let $\mathbf{M}^{*}$ be a dual translation surface of Type 1 with dual constant mean curvature $\mathbf{H}^{*} \neq 0$ in the three dimensional simply isotropic space $\rrbracket_{3}^{1}$. Then $f$ and $g$ are given by (15). 
Corollary 4.2. Let $\mathbf{M}^{*}$ be dual translation surface of Type 1 with zero mean curvature (isotropic minimal $\mathbf{H}^{*}=0$ ) in the three dimensional simply isotropic space $\square_{3}^{1}$. Then $f$ and $g$ are given by (9) $\operatorname{or}(10) .[5]$

Now suppose the dual isotropic Gaussian curvature $\mathbf{K}^{*}$ is a non-zero constant.

From (3), we have

$$
f^{\prime \prime}=\text { constant } \neq 0 \text { and } g^{\prime \prime}=\text { constant } \neq 0 \text {. }
$$

Thus, we have

$$
\left\{\begin{array}{l}
f(u)=c_{1} u^{2}+c_{2} u+c_{3}, \\
g(v)=c_{4} v^{2}+c_{5} v+c_{6},
\end{array}\right.
$$

where $c_{i} \in \mathbb{R}$.

Theorem 4.3. Let $\mathbf{M}^{*}$ be a dual translation surface of Type 1 with dual constant Gaussian curvature $\mathbf{K}^{*} \neq 0$ in the three dimensional simply isotropic space $\square_{3}^{1}$. Then $f$ and $g$ are given by (16).

Corollary 4.4. Let $\mathbf{M}^{*}$ be a dual translation surface of Type 1. Then, there is no isotropic flat $\left(\mathbf{K}^{*}=0\right)$ dual translation surface of Type 1 .

\section{Dual Isotropic Curvatures of The Dual Translation Surfaces of Type 2}

Suppose dual mean curvature $\mathbf{H}^{*}$ of the dual translation surface of Type 2 in $\mathbb{1}_{3}^{1}$ vanishes identically, from (5) we have

$$
f^{\prime \prime}(u) g^{\prime^{3}}(v)+g^{\prime}(v) g^{\prime \prime}(v)\left(1+f^{\prime^{2}}(u)\right)=0 .
$$

The above equation can be written as

$$
\frac{f^{\prime \prime}}{1+f^{\prime 2}}+\frac{g^{\prime \prime}}{g^{\prime 2}}=0
$$

Here $u$ and $v$ are independent variables, so each side of (17) is equal to a constant, call it $p$. Hence, the two equations are

$$
\frac{f^{\prime \prime}}{1+f^{\prime 2}}=p=-\frac{g^{\prime \prime}}{g^{\prime^{2}}} .
$$

Thus we get

$$
\left\{\begin{array}{l}
f(u)=c_{1}-\frac{\ln \left(\cos \left(p u-c_{2}\right)\right)}{p} \\
g(v)=c_{3}+\frac{\ln \left(p v-c_{4}\right)}{p}
\end{array}\right.
$$


where $p, c_{i} \in \mathbb{R}$. In particular, if $p=0$, from (18), we have

$$
\left\{\begin{array}{l}
f(u)=c_{1} u+c_{2} \\
g(v)=c_{3} v+c_{4}
\end{array}\right.
$$

Now, suppose dual mean curvature $\mathbf{H}^{*}$ of the dual translation surface of Type 2 in $\square_{3}^{1}$ is a nonzero constant, from (5), we have

$$
\frac{g^{\prime 3}}{g^{\prime \prime}}+\frac{g^{\prime}}{f^{\prime \prime}}\left(1+f^{\prime 2}\right)=2 \mathbf{H}^{*}
$$

Above equation can be rearranged in the form

$$
\frac{1+f^{\prime 2}}{f^{\prime \prime}}=\frac{2 \mathbf{H}^{*}}{g^{\prime}}-\frac{g^{\prime 2}}{g^{\prime \prime}} .
$$

Since $f$ and $g$ are functions of two independent variables, from (21), we have

$$
\frac{g^{\prime 2}}{g^{\prime \prime}}-\frac{2 \mathbf{H}^{*}}{g^{\prime}}=p=\frac{1+f^{\prime 2}}{f^{\prime \prime}}
$$

Thus, we get

$$
\left\{\begin{array}{l}
f=c_{2}-p \ln \left(\cos \left(\frac{u}{p}+c_{1}\right)\right) \\
g= \pm \sqrt{p^{2}-4 \mathbf{H}^{*}\left(v+c_{1}\right)}+c_{2}-p \ln \left( \pm p+\sqrt{p^{2}-4 \mathbf{H}^{*}\left(v+c_{1}\right)}\right)
\end{array}\right.
$$

where $p, c_{i} \in \mathbb{R}$.

Theorem 5.1. Let $\mathbf{M}^{*}$ be a dual translation surface of Type 2 with dual constant mean curvature $\mathbf{H}^{*} \neq 0$ in the three dimensional simply isotropic space $\square_{3}^{1}$. Then $f$ and $g$ are given by (22).

Corollary 5.2. Let $\mathbf{M}^{*}$ be dual translation surface of Type 2 with zero mean curvature (isotropic minimal $\mathbf{H}^{*}=0$ ) in the three dimensional simply isotropic space $\square_{3}^{1}$. Then $f$ and $g$ are given by (19) or (20). [5]

Now, if the dual isotropic Gaussian curvature $\mathbf{K}^{*}$ is constant, then from (5), we have

$$
f^{\prime \prime} \neq 0=\text { constant and } g^{\prime \prime} \neq 0=\text { constant. }
$$

Thus, we have

$$
\left\{\begin{array}{l}
f(u)=c_{1} u^{2}+c_{2} u+c_{3} \\
g(v)=c_{4} v^{2}+c_{5} v+c_{6}
\end{array}\right.
$$

where $c_{i} \in \mathbb{R}$. 
Theorem 5.3. Let $\mathbf{M}^{*}$ be a dual translation surface of Type 2 with dual constant Gaussian curvature $\mathbf{K}^{*} \neq 0$ in the three dimensional simply isotropic space $\square_{3}^{1}$. Then $f$ and $g$ are given by (23).

Corollary 5.4. Let $\mathbf{M}^{*}$ be a dual translation surface of Type 2. Then, there is no isotropic flat $\left(\mathbf{K}^{*}=0\right)$ dual translation surface of Type 2 .

\section{Dual Isotropic Curvatures of The Dual Translation Surfaces of Type 3}

If the dual mean curvature $\mathbf{H}^{*}$ of the dual translation surface of type 3 in $\rrbracket_{3}^{1}$ vanishes identically, from (8), we have

$$
\left(f^{\prime}+g^{\prime}\right)\left(f^{\prime \prime}\left(1+g^{\prime 2}\right)+g^{\prime \prime}\left(1+f^{\prime 2}\right)\right)=0 .
$$

We know that, in case of translation surface of type $3, f^{\prime}+g^{\prime} \neq 0$.

Thus, from (24), we have

$$
f^{\prime \prime}\left(1+g^{\prime 2}\right)+g^{\prime \prime}\left(1+f^{\prime 2}\right)=0
$$

The above equation can be written as

$$
\frac{f^{\prime \prime}}{1+f^{\prime 2}}+\frac{g^{\prime \prime}}{1+g^{\prime 2}}=0
$$

Since $u$ and $v$ are independent variables, from (25), we have

$$
\frac{f^{\prime \prime}}{1+f^{\prime 2}}=p=-\frac{g^{\prime \prime}}{1+g^{\prime 2}} \text {. }
$$

Thus, we get

$$
\left\{\begin{array}{l}
f=c_{1}-\frac{\ln \left(\cos \left(p u+c_{2}\right)\right)}{p}, \\
g=c_{3}+\frac{\ln \left(\cos \left(p v-c_{4}\right)\right)}{p},
\end{array}\right.
$$

where $p, c_{i} \in \mathbb{R}$. In particular if $p=0$, from (26), we have

$$
\left\{\begin{array}{l}
f=c_{1} u+c_{2} \\
g=c_{3} v+c_{4}
\end{array}\right.
$$

Now, considering dual translation surface of Type 3 with non-zero dual constant mean curvature in simply isotropic 3 -space $\mathbb{1}_{3}^{1}$. If the mean curvature $\mathbf{H}^{*}$ is non-zero constant, from (8), we have

$$
\frac{\left(f^{\prime}(u)+g^{\prime}(v)\right)\left(f^{\prime \prime}(u)\left(1+g^{\prime^{2}}(v)\right)+g^{\prime \prime}(v)\left(1+f^{\prime^{2}}(u)\right)\right)}{16 f^{\prime \prime}(u) g^{\prime \prime}(v)}=c .
$$


Above equation can be rearranged as

$$
\left(f^{\prime}+g^{\prime}\right)\left(\frac{\left(1+g^{\prime 2}\right)}{g^{\prime \prime}}+\frac{\left(1+f^{\prime 2}\right)}{f^{\prime \prime}}\right)=16 c .
$$

Differentiating (29), w.r.t. $u$ and $v$, we get

$$
f^{\prime \prime}\left(\frac{1+g^{\prime 2}}{g^{\prime \prime}}\right)^{\prime}+g^{\prime \prime}\left(\frac{1+f^{\prime 2}}{f^{\prime \prime}}\right)^{\prime}=0
$$

or,

$$
\frac{1}{g^{\prime \prime}}\left(\frac{1+g^{\prime 2}}{g^{\prime \prime}}\right)^{\prime}+\frac{1}{f^{\prime \prime}}\left(\frac{1+f^{\prime 2}}{f^{\prime \prime}}\right)^{\prime}=0 .
$$

Since $u$ and $v$ are independent variables, from (30), we get

$$
\left(\frac{1+g^{\prime 2}}{g^{\prime \prime}}\right)^{\prime}=p g^{\prime \prime}
$$

and

$$
\left(\frac{1+f^{\prime 2}}{f^{\prime \prime}}\right)^{\prime}=-p f^{\prime \prime}
$$

Integrating (31), we get

$$
\frac{1+g^{\prime 2}}{g^{\prime \prime}}=p g^{\prime}
$$

Using change of variables, $g^{\prime}=t$, from (33), we have

$$
\frac{1}{t}+t=p t^{\prime}
$$

Thus, we get

$$
t= \pm \sqrt{-1+e^{\frac{2}{p}\left(v+c_{1}\right)}} .
$$

Integrating again for $g$, we obtain

$$
g= \pm p\left(\sqrt{-1+e^{\frac{2}{p}\left(v+c_{1}\right)}}-\tan ^{-1}\left(\sqrt{-1+e^{\frac{2}{p}\left(v+c_{1}\right)}}\right)\right) .
$$

Similarly from (32), we can easily obtain

$$
f= \pm p\left(e^{\frac{-u}{p}} \sqrt{-e^{\frac{2 u}{p}}+e^{2 c_{1}}}+\tan ^{-1}\left(\frac{e^{\frac{u}{p}}}{\sqrt{-e^{\frac{2 u}{p}}+e^{2 c_{1}}}}\right)\right) .
$$

In particular if $p=0$, from (31) and (32), we get

$$
\left\{\begin{array}{l}
f=-u+c e^{\frac{u}{c}} c_{1}+c_{2}, \\
g=-v+c e^{\frac{v}{c}} c_{3}+c_{4},
\end{array}\right.
$$

where $c_{i} \in \mathbb{R}$. 
Theorem 6.1. Let $\mathbf{M}^{*}$ be dual translation surface of Type 3 with zero mean curvature (isotropic minimal $\mathbf{H}^{*}=0$ ) in the three dimensional simply isotropic space $\square_{3}^{1}$. Then $f$ and $g$ are given by (27) or (28).

Theorem 6.2. Let $\mathbf{M}^{*}$ be a dual translation surface of Type 3 with dual constant mean curvature $\mathbf{H}^{*} \neq 0$ in the three dimensional simply isotropic space $\square_{3}^{1}$. Then $f$ and $g$ are given by (34) and (35) or (36).

Now, if the dual isotropic Gaussian curvature $\mathbf{K}^{*}$ vanishes, then from (7), we have

$$
\frac{\left(f^{\prime}+g^{\prime}\right)^{4}}{16 f^{\prime \prime} g^{\prime \prime}}=0 \text {. }
$$

Thus, $f$ and $g$ must be constants.

If the dual isotropic Gaussian curvature $\mathbf{K}^{*}$ is constant, then from (7), we have

$$
\frac{\left(f^{\prime}+g^{\prime}\right)^{4}}{16 f^{\prime \prime} g^{\prime \prime}}=\mathbf{K}^{*} \text {. }
$$

The above equation can be written as

$$
f^{\prime}+g^{\prime}=2 \mathbf{K}^{*}\left(f^{\prime \prime} g^{\prime \prime}\right)^{\frac{1}{4}}
$$

Differentiating (37), w.r.t. u, we get

$$
\frac{\left(f^{\prime \prime}\right)^{\frac{7}{4}}}{f^{\prime \prime \prime}}=\frac{\mathbf{K}^{*}}{2} g^{\prime \prime} .
$$

Since, $f$ and $g$ are the functions of two independent variables, we have

$$
\frac{\left(f^{\prime \prime}\right)^{\frac{7}{4}}}{f^{\prime \prime \prime}}=p=-\frac{\mathbf{K}^{*}}{2} g^{\prime \prime}, \text { where } p \in \mathbb{R} .
$$

Thus, we get

$$
\left\{\begin{array}{l}
f=-2 p^{2}\left(\frac{-u}{p}+c_{1}\right)^{\frac{2}{3}} 6^{\frac{2}{3}}+c_{2} u+c_{3}, \\
g=-\frac{p}{\mathbf{K}^{*}} v^{2}+c_{4} v+c_{5} .
\end{array}\right.
$$

In particular if $p=0$, from (39), we have

$$
\left\{\begin{array}{l}
f=c_{1} u+c_{2} \\
g=c_{3} v+c_{4}
\end{array}\right.
$$

where $c_{i} \in \mathbb{R}$.

Theorem 6.3. Let $\mathbf{M}^{*}$ be a dual translation surface of Type 3 with dual constant Gaussian curvature $\mathbf{K}^{*} \neq 0$ in the three dimensional simply isotropic space $\square_{3}^{1}$. Then $f$ and $g$ are given by (40) or (41).

Corollary 6.4. Let $\mathbf{M}^{*}$ be a dual translation surface of Type 3 with dual constant Gaussian curvature $\mathbf{K}^{*}=0$ in the three dimensional simply isotropic space $\square_{3}^{1}$. Then $f$ and $g$ are constants. 


\section{Acknowledgement}

The authors are very grateful to the anonymous referees for the time they dedicated to this article and for the comments they made.

\section{References}

[1] M. E. Aydin, A generalization of translation surfaces with constant curvature in the isotropic space, J. Geom., 107(2016), 603-615.

[2] B. Bukcu, D. W. Yoon and M. K. Karacan, Translation surfaces in the 3-dimensional simply isotropic space $\square_{3}^{1}$ satisfying $\Delta^{I I I} x_{i}=\lambda_{i} x_{i}$, Konuralp Journal of Mathematics, 4 (2016), 275-281.

[3] M. K. Karacan, D. W. Yoon and B. Bukcu, Translation surfaces in the three dimensional simply isotropic space $\square_{3}^{1}$, Int. J. Geom. Methods Mod. Phys., 13 (2016), 1650088.

[4] M. K. Karacan and N. Yuksel, Translation Surfaces of Type 3 in the Three Dimensional Simply Isotropic Space, submitted

[5] M. K. Karacan, N. Yuksel, A. Cakmak and S. Kizltug, Dual Surfaces Defined by $z=f(u)+g(v)$ in Simply Isotropic 3-Space, submitted.

[6] H. Pottmann, P. Grohs and N. J. Mitra, Laguerre minimal surfaces, isotropic geometry and linear elasticity, Adv. Comput. Math., (2009), 31:391.

[7] H. Pottmann and Y. Liu, Discrete Surfaces in Isotropic Geometry, Mathematics of Surfaces XII,Volume 4647 of the series Lecture Notes in Computer Science, (2007), 341-363.

[8] H. Sachs, Isotrope Geometrie des Raumes, Vieweg Verlag, Braunschweig, 1990.

[9] Z. M. Sipus, Translation Surfaces of constant curvatures in a simply Isotropic space, Period Math. Hung., 68 (2014), 160-175.

[10] K. Strubecker, Differentialgeometrie des Isotropen raumes III, Flachentheorie, Math. Zeitsch., 48 (1942), 369 427.

[11] K. Strubecker, Duale Minimalflachen des isotropen Raumes, Rad JAZU, 382 (1978), 91-107.

[12] D. W. Yoon and J. W. Lee, Linear Weingarten Helicoidal Surfaces in Isotropic Space, Symmetry 2016, 8, 126; doi:10.3390/sym8110126

Department of Mathematics, Central University of Jammu, J\& K, 180011, India.

E-mail: saleemraja2008@gmail.com

Usak University, Faculty of Sciences and Arts, Department of Mathematics, 1 Eylul Campus, 64200, Usak-TURKEY.

E-mail: murat.karacan@usak.edu.tr 